\title{
Multivariate analysis to predict letrozole efficacy in improving sperm count of non-obstructive azoospermic and cryptozoospermic patients: a pilot study
}

\author{
Giorgio Cavallini ${ }^{1}$, Giulio Biagiotti ${ }^{2}$ and Elisa Bolzon ${ }^{1}$
}

We tested the hypothesis that letrozole increases sperm count in non-obstructive azoospermic or cryptozoospermic patients with a testosterone (T)/17-beta-2-oestradiol (E2) ratio $<10$. Forty-six patients with no chromosomal aberrations were randomized into two groups: 22 received letrozole $2.5 \mathrm{mg}$ per day for 6 months (Group 1: 6 azoospermic +16 cryptozoospermic patients), while 24 received a placebo (Group 2: 5 azoospermic + 19 cryptozoospermic patients). The following data were collected: two semen analyses, clinical history, scrotal Duplex scans, body mass index (BMI), Y microdeletion, karyotype and cystic fibrosis screens and follicle-stimulating hormone (FSH), luteinizing hormone (LH), E2, T and prolactin levels. Both before and after letrozole or placebo administration, the patients underwent two semen analyses and hormonal assessments. The differences were evaluated using the Mann-Whitney $U$ test. The relationships between sperm concentration after letrozole administration with respect to FSH, T/E2 ratio, bilateral testicle volume and BMI before letrozole administration were assessed using multivariate analysis. The side effects were assessed using the chi-square test. Group 1 had sperm concentration (medians: $400-1.290 \times 10^{6} \mathrm{ml}^{-1} ; P<0.01$ ) and motility (medians: class A from $2 \%$ to $15 \%$; $\boldsymbol{P}<0.01$ ), FSH, LH and T significantly increased, while Group 2 did not. E2 levels diminished significantly in Group 1, but not in Group 2. Eight patients in Group 1 demonstrated side effects, whereas no patient side effects were observed in Group 2 . The sperm concentration after letrozole administration is inversely related to T/E2, FSH and BMI; a direct relationship emerged between sperm concentration and testicular volume.

Asian Journal of Andrology (2013) 15, 806-811; doi:10.1038/aja.2013.99; published online 14 October 2013

Keywords: cryptozoospermia; letrozole; male infertility; non-obstructive azoospermia

\section{INTRODUCTION}

Idiopathic (oligo-) asthenoteratozoospermia (iOAT) is defined as a type of impaired spermatogenesis in which sperm production is altered, but the common clinical and laboratory parameters are within the normal range. iOAT affects approximately $30 \%$ of infertile men. Therefore, iOAT patients may be considered a heterogeneous cohort of infertile men. ${ }^{1}$ Idiopathic patients with a low $(<10)$ testosterone (T)/oestradiol (E2) ratio exhibited an increased sperm count after letrozole and/or anastrozole treatment. ${ }^{2,3}$ Non-obstructive azoospermic (NOA) patients with $\mathrm{T} / \mathrm{E} 2<10$ also had their sperm count increased with letrozole and/or anastrozole treatment. ${ }^{4-6}$ Letrozole and anastrozole are members of a novel class of non-steroidal, hormone-targeting agents used for breast cancer therapy. They reversibly inhibit the aromatase enzyme, which converts the androgen precursors in adipose tissue to E2. Blocking of oestrogen production has been shown to provoke increased gonadotropin and androgen levels in the blood and a parallel E2 decrease, resulting in spermatogenesis stimulation. $^{2-5}$

Our team has previously used letrozole; ${ }^{4}$ however, a placebo-controlled study examining the efficacy and safety of letrozole is lacking, and it is unknown whether letrozole efficacy might be influenced by clinical spermatogenesis markers such as baseline follicle-stimulating hormone (FSH), testicular volume and body mass index (BMI). The current pilot prospective, double-blind, controlled study tested the hypothesis that letrozole administration to NOA or to cryptozoospermic patients with $\mathrm{T} / \mathrm{E} 2<10$ could increase their sperm count more significantly than a placebo in relation to baseline $\mathrm{FSH}$, testicular volume and BMI.

\section{MATERIALS AND METHODS}

This was a pilot, multicentre, randomized, double-blind, placebocontrolled study, and was authorized by the Institutional Review Board of Andros-Italia. Patient recruitment began on 2 January 2010 and finished on 31 March 2012, and written informed consent was obtained.

Patients

The patients were randomly assigned to one of two groups to receive either letrozole $2.5 \mathrm{mg}$ per day or a placebo (starch). The Data Monitoring Committee reviewed the unblinded data for patient safety; there were no interim analyses for efficacy. Non-smoker NOA patients who yielded no spermatozoa with fine needle aspiration

${ }^{1}$ Andros-Italia, Outpatient Clinic of Ferrara, Ferrara 44121, Italy and ${ }^{2}$ Andros-Italia, Outpatient Clinic of Perugia, Perugia 06128, Italy Correspondence: Dr G Cavallini (giorgiocavallini@libero.it)

Received: 17 March 2013; Revised: 22 May 2013; Accepted: 12 July 2013; Published online: 14 October 2013 
$(\text { FNA })^{7}$ and cryptozoospermic patients with T/E2 ratio $<10$ were considered as candidates for inclusion in the study. Azoospermia was defined as the absence of sperm in the pellets of two centrifuged (300 $\mathrm{g}$ for $15 \mathrm{~min}$ ) semen samples collected 7-30 days apart; cryptozoospermia was defined as the presence of sperm in the pellet (but not in the ejaculate) of at least one semen sample out of the two collected, i.e., with a sperm concentration $<10^{3} \mathrm{ml}^{-1.8}$.

Eligible patients were those with normal sperm appearance, consistency, liquefaction, volume, and $\mathrm{pH}$ and no chromosomal aberrations. One hundred and thirty patients were assessed for eligibility, 30 of whom refused to participate (Figure 1). The patients were not admitted to the study if any of the following criteria were present: seminal white blood cell concentration greater than $10^{6} \mathrm{ml}$ and/or a positive seminal cultural analysis or positive urethral swab chlamydia test (16 patients); drug, tobacco, or alcohol abuse (8 patients); ongoing medical treatment (gonadotropins, anabolic steroids, cancer chemotherapy, non-steroidal anti-inflammatory drugs (3 patients); previous cancer radiotherapy or chemotherapy (3 patients), palpable varicocele (10 patients); $\mathrm{X}$-ray exposure in the previous 8 months (3 patients); $\mathrm{Y}$ chromosome microdeletion (3 cases), and karyotype alterations (2 cases of Klinefelter syndrome) (Figure 1).

Clinical history, physical examination, bilateral scrotal Duplex scans, blood hormonal levels of FSH, luteinizing hormone (LH), E2, $\mathrm{T}$ and prolactin (PRL), and genetic assessment (karyotype, $\mathrm{Y}$ microdeletion and cystic fibrosis screening) were performed on each patient. Testicular volume $\left(\mathrm{cm}^{3}\right)$ was measured by ultrasonography using the three diameter technique (length $\times$ width $\times$ height $\times 0.71$ ). A differential diagnosis between obstructive azoospermia and NOA patients

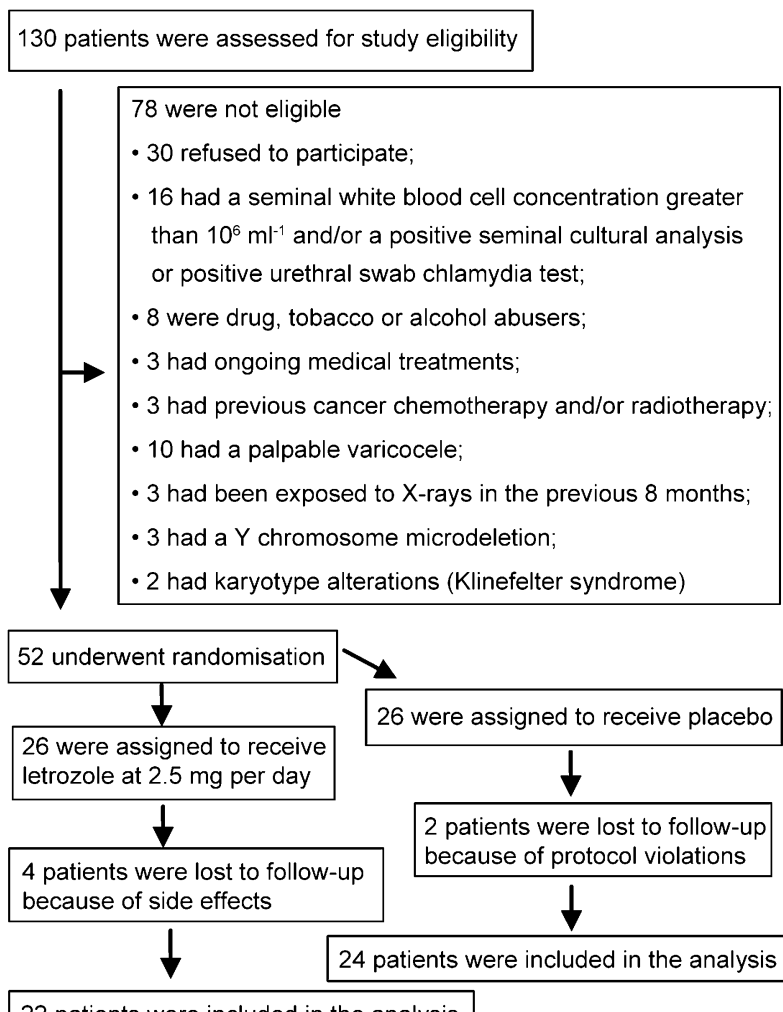

22 patients were included in the analysis

Figure 1 Flow chart of a multicenter trial of letrozole $2.5 \mathrm{mg}$ per day for 6 months vs. placebo for improving the symptoms in non-obstructive azoospermic and cryptozoospermic patients. The chart includes detailed information regarding the patients excluded. was determined using FNA. ${ }^{7}$ All cryptozoospermic patients had their semen cryoconserved before active drug or placebo administration.

The patients were randomly assigned to two groups to receive the active drug (Group 1) (letrozole (Femara; Aventis Pharma, Mumbai, India) $2.5 \mathrm{mg}$ once a day) or a placebo (Group 2) (starch $100 \mathrm{mg}$ once a day) for 6 months. The following assessments were performed before the 3 and 6 months after drug administration: two sperm analyses and FSH, LH, E2, T and PRL measurements.

\section{Semen examination}

Each patient provided two semen samples following masturbation; each sample was given a code number. No spermatozoa could be found in ejaculated semen samples before treatment in both the letrozole- and placebo-treated patients; the samples were centrifuged at $300 \mathrm{~g}$ for $15 \mathrm{~min}$. The pellet was gently resuspended in $100 \mu \mathrm{l}$ of culture medium (Ham's F-10 supplemented with $10 \%$ bovine serum albumin medium; Sigma-Aldrich, St Louis, MO, USA); sperm concentration and motility were assessed using an improved double-rule Neubauer haemocytometer without any sample dilution. The entire semen preparation was examined, and all spermatozoa were counted and assessed for motility under $\times 10$ and $\times 40$ magnifications. ${ }^{9}$ The morphology assessment requires Papanicolaou staining (i.e., sample fixation); therefore, the morphology could not be assessed in these cases because of the extremely low number of spermatozoa. ${ }^{9}$

The sperm concentration increased in the active drug group after letrozole administration; thus, a 1:10 dilution of the semen sample was prepared with a glass tuberculin syringe using Ham's F-10 supplemented with $10 \%$ bovine serum albumin medium. An improved double-rule Neubauer haemocytometer was used to count the spermatozoa. Two dilutions of each semen sample were prepared. The difference between the two dilutions was not greater than $10 \% .{ }^{9}$ A motility assessment was performed on two samples from the same ejaculate observing at least 200 spermatozoa in each and using at least 10 randomly selected, unique high-power microscopic fields. The motility of each sperm was graded A, B or C according to whether it showed (A) progressive motility, (B) non-progressive motility or (C) immotility. ${ }^{9}$ Each ejaculate was evaluated for sample variability using Pearson's coefficient correlation analysis. Because values $>0.90$ and $<0.93$ were obtained, the differences between the two samples did not require any further analyses. ${ }^{10}$ To assess sperm morphology, two semen smears from each patient were stained at the laboratory using the Papanicolaou method and assessed for sperm morphology by one technician. A total of 200 or 300 sperm were analysed per slide. Initially, 200 sperms from each of two different locations on the slide were analysed. The mean sperm value was calculated if the difference between the percentages of normal sperm in the two areas tested was five percentage points or fewer. An additional 200 sperm were evaluated from a third location on the slide, and the median of all three values was used if the difference was more than five percentage points. ${ }^{9,11}$

\section{End points and statistical analysis}

This was a pilot study; therefore, a power analysis was not performed. ${ }^{10}$ The primary end point with respect to the letrozole efficacy was an increase in sperm concentration and motility. Secondary end points included differences in FSH, LH, E2, T and PRL levels after active drug or placebo administration. The differences between the levels before and after therapy were assessed using the Mann-Whitney $U$ test and were corrected for the number of samples delivered (two samples). ${ }^{10}$ Additional secondary end points were the relationships 
between sperm concentration and motility after 6 months of letrozole administration with respect to FSH and T/E2 levels, and bilateral testicular volume and BMI before letrozole administration. These end points were assessed using non-parametric Spearman's rank analysis and were corrected for the number of sperm samples (2) delivered by each patient. ${ }^{10}$ The levels of significance maintained an overall $P$ value of 0.05 and were calculated according to the O'Brien-Fleming stopping boundary for the primary end points and the Pocock boundary for the secondary end points. ${ }^{12}$

\section{Procedures to ensure blindness and randomisation}

Randomisation of the samples was performed using casual number tables. ${ }^{10}$ The blindness for drug delivery was ensured by using colourcoded boxes, and the blindness of seminal examination was ensured by assigning each sample with a code number. The nurses were blinded to the delivery of the boxes, and the biologists were blinded to the seminal examinations performed. All of the study personnel and participants were blinded to the treatment assignment for the duration of the study. Only the Andros-Italia Institutional Review Board saw the unblinded data in order to alert physicians in case of major side effects, but this action was never necessary, and no one from the Institutional Review Board had any contact with the study participants. Colour and numerical codes were disclosed at the end of the study. ${ }^{13}$

\section{RESULTS}

Fifty-two patients were eligible for participation in the study: 26 were randomly assigned to receive the active drug (Group 1: letrozole $2.5 \mathrm{mg}$ per day) and 26 the placebo (Group 2). Four patients dropped out of Group 1 due to side effects (loss of libido: two cases; loss of hair: one case; and cutaneous rash: one case). Thus, 22 Group 1 patients were examined. Two patients were eliminated from Group 2 because of protocol violations; thus, 24 Group 2 patients were studied (Figure 1).

Table 1 shows the baseline demographic and clinical characteristics of each group. The differences between the treated and the placebo groups were assayed using the chi-square test (differences between percentage values) or the Mann-Whitney $U$ test (differences between the medians). ${ }^{10}$ No significant differences were observed.

Table 2 summarizes the results of each study group: i.e., sperm concentration and motility and FSH, LH, PRL, T and E2 levels before and then after a 3 - and 6-month administration of letrozole $2.5 \mathrm{mg}$ per day (Group 1) or a placebo (Group 2). Sperm concentration, sperm motility, FSH, LH and T significantly increased in Group 1 patients at 3 and 6 months (there was no significant difference between the 3 - and the 6-month data), but no improvements were observed in Group 2 patients. Conversely, E2 levels were significantly decreased in Group 1 patients at 3 and 6 months (there was no significant difference between the 3- and 6-month data), but no significant difference was demonstrated in Group 2 patients. No natural pregnancies occurred in either group. No significant modification in PRL levels occurred in either Group 1 or Group 2 patients. The side effects were significantly higher in the group of patients treated with the active drug. Five patients demonstrated loss of libido and hair, two patients had cutaneous rashes and one patient reported only a loss of libido. Spermatozoa could be found in the ejaculate of all NOA patients treated with letrozole, while the NOA patients treated with the placebo remained azoospermic.

The results of the multivariate analysis to assess the relationships between sperm concentration, after active drug administration and FSH level, T/E2 ratio, bilateral testicular volume and BMI, were indicated by the following equation where: $Y$ represents the expected concentration of spermatozoa per $\mathrm{ml}$ after letrozole administration, $X_{1}$ represents the T/E2 ratio; $X_{2}$ the FSH baseline blood level before letrozole administration; $X_{3}$ the bilateral testicular volume before letrozole administration and $X_{4}$ the BMI before active drug administration: $Y=0.1-3.2 X_{1}-4.0 X_{2}+4.6 X_{3}+4.7 X_{4}$. Thus, the sperm concentration after letrozole administration was significantly and inversely related to the T/E2 ratio $\left(t_{1}=13.124, P<0.01\right)$ and $\mathrm{FSH}$ $\left(t_{2}=7.976, P<0.05\right)$, whereas sperm concentration was significantly and directly linked to bilateral testicular volume $\left(t_{3}=7.200, P<0.05\right)$ and to BMI $\left(t_{4}=6.265, P<0.05\right)$. The T/E2 ratio is the most important element for increased sperm count; FSH, testicular volume and BMI were less important.

\section{DISCUSSION}

These data show that letrozole increases the sperm count of NOA or cryptozoospermic patients. Spermatozoa were found in the ejaculate of all the NOA patients treated with letrozole, while no spermatozoa could be found in the ejaculate of the NOA patients treated with placebo. Increases in sperm counts occurred after a 3-month administration, and no significant difference was observed between the

Table 1 Baseline demographic and clinical characteristics for each group.

\begin{tabular}{|c|c|c|}
\hline & $\begin{array}{c}\text { Group 1: letrozole } \\
\qquad(n=21)\end{array}$ & $\begin{array}{l}\text { Group 2: placebo } \\
\qquad(\mathrm{n}=22)\end{array}$ \\
\hline Age (year) ${ }^{a}$ & $44(37-52)$ & $45(38-53)$ \\
\hline $\mathrm{FSH}\left(I U \mathrm{I}^{-1}\right)^{\mathrm{a}}$ & $14(6.5-25)$ & $15(5.0-27.2)$ \\
\hline $\mathrm{LH}\left(\mid \mathrm{I} \mathrm{I}^{-1}\right)^{\mathrm{a}}$ & $4.2(2.3-9.2)$ & $3.9(2.1-9.6)$ \\
\hline$T\left(n g d^{-1}\right)^{a}$ & $249.0(200.3-298.1)$ & $266.2(212.0-312.3)$ \\
\hline $\mathrm{E} 2\left(\mathrm{pg} \mathrm{ml^{-1 }}\right)^{\mathrm{a}}$ & $44.4(40.2-47.3)$ & $43.7(41.3-48.9)$ \\
\hline $\mathrm{PRL}\left(\mathrm{ng} \mathrm{ml}^{-1}\right)^{\mathrm{a}}$ & $14.3(9.0-27.2)$ & $15.0(10.0-28.3)$ \\
\hline Bilateral testicular volume $\left(\mathrm{cm}^{3}\right)^{a}$ & $16.7(10.3-21.7)$ & $18.4(11.9-23.7)$ \\
\hline Body mass index $\left(\mathrm{kg} \mathrm{m}^{-2}\right)^{\mathrm{a}}$ & $31.2(27.2-36.5)$ & $30.7(23.6-36.8)$ \\
\hline Azoospermic patients, $n(\%)$ & $6(27.3 \%)$ & $5(20.8 \%)$ \\
\hline Cryptozoospermic patients, $n(\%)$ & $16(72.7 \%)$ & $19(79.2 \%)$ \\
\hline Previous bilateral cryptorchidism, $n(\%)$ & $3(13.6 \%)$ & $4(16.7 \%)$ \\
\hline Previous unilateral cryptorchism, $n(\%)$ & $5(22.7 \%)$ & $5(20.8 \%)$ \\
\hline Patients without previous identifiable cause of azoospermia or cryptozoospermia, $n(\%)$ & $14(63.6 \%)$ & $15(62.5 \%)$ \\
\hline
\end{tabular}

Abbreviations: E2, 17-beta-2-oestradiol; FSH, follicle-stimulating hormone; LH, luteinizing hormone; PRL, prolactin; T, total testosterone.

${ }^{a}$ Data are shown as median values and ranges are in parentheses.

All differences between the comparisons were not significant. 
3- and the 6-month administration regimen. No natural pregnancies occurred in either group. The efficacy of letrozole administration in increasing sperm count was linked primarily to the severity of T/E2 ratio impairment and, to a lesser extent, to $\mathrm{BMI}$ impairment, testicular volume and FSH (i.e., the lower the T/E2 ratio and $\mathrm{FSH} /$ the higher BMI and testicle volume, the higher the sperm count is after active drug administration). Hormonal differences between pre- and postletrozole administration agree with previous literature, which indicated that letrozole increases sperm count by increasing gonadotrophin, T/E2 and testosterone levels. ${ }^{2-4}$ Because the literature is in agreement with the data presented here, we can confirm that letrozole increased the sperm count in dispermic patients with T/E2 $<10$.

Raman and Schlegel ${ }^{3}$ evaluated the effect of anastrozole on the hormonal and semen parameters of 104 infertile men who had an abnormal T/E2 ratio. However, those authors included patients with Klinefelter syndrome and varicoceles; in that study, only 25 patients had iOAT, and a placebo-untreated group was not included. The patients demonstrated increased sperm count and T/E2 level after treatment, and pregnancies were not reported. ${ }^{3}$ Patry et al. ${ }^{5}$ reported one NOA case of hypospermatogenesis, which was converted to active spermatogenesis after a 3-month regimen of letrozole. However, the patient remained azoospermic due to an obstruction in the epididymis that was not adequately assessed when surgery was performed. ${ }^{5}$ Gregoriou et al. ${ }^{2}$ described 15 patients who were treated with $2.5 \mathrm{mg}$ letrozole per day and 14 patients who were treated with $1 \mathrm{mg}$ anastrozole per day ${ }^{3}$ for 6 months in a poorly controlled clinical study (alternated allocation of enrolled patients without a placebo or an untreated group) in which all patients had T/E2 $<10$. All patients also had an increased sperm count and a T/E2 ratio. $^{2}$ Saylam et al..$^{6}$ treated a total of 27 infertile male patients ( 10 were iOAT and 17 were NOA) who had low serum testosterone levels and T/E2 ratios $<10$ using letrozole $2.5 \mathrm{~g}$ per day for 6 months. Four NOA patients demonstrated that the sperm return to the ejaculate after treatment, all iOAT patients demonstrated an increased sperm count, and two fathered children. The T levels had increased in all cases. ${ }^{6}$ A follow-up study by our group reported on four NOA patients with FSH levels $<10$ UI $1^{-1}$ who all had sperm counts increase in the ejaculate during letrozole therapy $(2.5 \mathrm{mg}$ per day for 3 months). FSH and T strongly increased in all cases. ${ }^{4}$

Although all investigators described a sperm count increase after letrozole/anastrozole treatment and similar increases of blood hormone levels, there are strong differences in the sperm count and in side effects between the different studies. These differences may indicate that individual differences in letrozole/anastrozole efficacy might occur, such as when this drug was used to treat breast cancers. ${ }^{14}$ In the latter case, the genetic polymorphisms of the metabolizing enzymes of the aromatase inhibitor as well as aromatase CYP19A1 functional genetic polymorphisms have been proposed to explain the individual differences of drug efficacy. ${ }^{14}$ Similar mechanisms may therefore occur in dyspermic patients. Surprisingly, six of our letrozole-treated patients reported a loss of libido, despite the supraphysiological levels of $\mathrm{T}$, and confirmed that oestrogens could play a role in supporting libido. $^{15}$

The following considerations compelled us to study the NOA patients who yielded no spermatozoa following FNA. Despite the availability of ultrasound and biochemical techniques to differentiate NOA patients from obstructive azoospermia patients, ${ }^{16-18}$ FNA has been regarded as a highly informative and minimally invasive method. ${ }^{7}$ Furthermore, the safety of FNA use was regarded as being supportive for the researchers against any possible patient lawsuits resulting from any impaired spermatogenesis induced by an off-label prescription. 
Intention-to-treat analysis has been used as the gold standard for estimating treatment effects in randomized trials. This was a blind placebo-controlled study in which the active drug was an 'off-label' prescription of letrozole; thus, we presumed low patient compliance (30 patients refused to participate) and a high proportion of patient dropouts, which occurred as soon as a minimal side effect occurred. NOA and cryptozoospermic patients with T/E2 <10 comprise approximately $30 \%$ of the entire population with this type of sperm alteration. These considerations compelled us to limit this research to a pilot study because a higher number of patients (i.e., as requested by power analysis) would have required excessive time-consuming research. The robustness of intention-to-treat analysis can be challenged in the presence of missing outcomes. Therefore, we preferred to form conclusions exclusively on data from patients who completed the follow-up: accordingly, the power of the analysis decreased, but improved the robustness of the analysis. ${ }^{19}$ For these reasons, early stopping rules were used (i.e., the O'Brien-Fleming and Pocock boundary) and the abovementioned patient samples were used. Thus, although this was a pilot study, the above considerations might have limited our research, at least in part, due to theoretical biases regarding the number of patients studied. Furthermore, the number of the patients studied is similar or even higher than that reported in previous studies. $^{2-6}$

Our data show that BMI and the T/E2 ratio were not strictly equivalent in predicting the results of active drug administration or spermatogenesis impairment, even though BMI is closely associated with the T/E2 ratio and spermatogenesis. ${ }^{20,21}$ Interestingly, these data were in agreement with the previous results of Raman and Schlegel, where obese patients did not have a greater benefit of treatment than other patients, even though aromatase from adipose tissue could increase aromatase activity. This observation may reflect the hypothesis that aromatase activity essentially comes from the testis and not from its peripheral conversion in other tissues. ${ }^{22}$ Furthermore, male obesity is associated with physiological changes, which could negatively affect male fertility. Obese men have a threefold increase in the prevalence of oligozoospermia when compared to men with a normal BMI. The proposed causes of reduced spermatogenesis include reduced circulating and intratesticular T levels and increased E2 levels. Increased E2 levels are postulated to contribute to the central suppression of gonadotrophin production, which in turn, may decrease both testosterone production and spermatogenesis. ${ }^{23} \mathrm{E} 2$ is derived mainly from the intratesticular and peripheral aromatisation of androstenedione and testosterone by aromatase, a CYP19 gene product. CYP19A1 is a single-copy gene located on chromosome 15q21.2. Aromatase polymorphisms have been shown to affect various oestrogen-dependent diseases in men and women. The most commonly studied aromatase polymorphism is the tetranucleotide tyrosine-tyrosine-tyrosineadenine (TTTA) repeat polymorphism (TTTAn) present in intron 4 of the CYP19A1 gene. This polymorphism is associated with the activity of the aromatase enzyme both in vivo and in vitro. ${ }^{24}$ Higher TTTA repeat numbers ( $>7$ repeats) in the aromatase gene are associated with a negative relationship between obesity and sperm count. The effect of obesity on E2 and sperm count appears to be absent in men with low $(\leqslant 7)$ TTTA repeats. ${ }^{20}$

FSH is considered as a marker of spermatogenesis and Sertoli cell function, and several studies have reported that the risk of abnormal semen parameters is negatively correlated to sperm concentration even for blood concentrations lower than the conventional upper limit of the reference interval of $10-12 \mathrm{IU}^{-1} \cdot{ }^{25,26}$ Particularly intriguing for our results was the recent demonstration that an increased risk of abnormal semen analyses occurs among men with FSH levels $>4.5 \mathrm{IU} \mathrm{l}^{-1}$ and lower testosterone/FSH ratios, ${ }^{26}$ confirming the pivotal role of testosterone levels in spermatogenesis. Similarly, the testicular volume is regarded as a clinical marker of spermatogenesis, which is negatively correlated with the reproductive activity of the testicle. ${ }^{16,27}$

The emerging picture is that less compromised spermatogenesis demonstrated an increased sperm count following letrozole treatment, when the T/E2 ratio is lower than 10. These data might explain, other than individual genetic differences (see above), why oligoasthenoteratospermic patients treated in the literature with aromatase inhibitors $^{2,3,6}$ displayed a higher sperm count after letrozole/anastrozole treatment than in our cases of NOA/cryptozoospermic patients. Attempts to administer letrozole to six patients with T/E2 $>10$ did not increase their sperm count.

Currently, it is unknown whether the success percentage of surgical sperm retrieval from the testicles of patients with a low T/E2 ratio may differ from the success obtained in patients with a normal T/E2 ratio. However, the finding of spermatozoa in the ejaculate of all NOA patients has saved patients from surgery and from the potential failure of surgical sperm retrieval, which may represent, other than an increased sperm count, additional clinical benefits of letrozole administration. The impact of an increased sperm count on the success of assisted reproduction techniques occurring after letrozole administration will be presented in a future study; however, because the quality of spermatogenesis in NOA patients is linked to intracytoplasmic sperm injection pregnancy rates, even mild improvement can improve the likelihood of pregnancy. ${ }^{28}$ This might be particularly interesting for Italians since their law limits the efficiency of assisted reproduction treatment in couples with a severe male factor. ${ }^{29,30}$

It is unlikely that letrozole increased congenital malformations, as no malformed babies were fathered during the course of letrozole treatment. ${ }^{6}$ Additional studies have also shown that letrozole does not increase the risk of congenital malformations with ovulation induction. ${ }^{31}$ In conclusion, letrozole should be considered a reliable resource for increasing the sperm count in NOA and cryptozoospermic patients with T/E2 $<10$.

\section{AUTHOR CONTRIBUTIONS}

GC and EB studied 10 patients treated with letrozole and 13 with placebo in the outpatient clinic of Ferrara; GB studied 12 patients treated with letrozole and 10 treated with placebo in the outpatient clinic of Perugia. GC wrote the manuscript; GB and EB revised and edited the manuscript.

\section{COMPETING FINANCIAL INTERESTS}

All authors declare no competing financial interests.

1 Cavallini G, Crippa A, Magli MC, Cavallini N, Ferraretti AP et al. A study to sustain the hypothesis of the multiple genesis of oligoasthenoteratospermia in human idiopathic infertile males. Biol Reprod 2008; 79: 667-73.

2 Gregoriou O, Bakas P, Grigoriadis C, Creatsa M, Hassiakos D et al. Changes in hormonal profile and seminal parameters with use of aromatase inhibitors in management of infertile men with low testosterone to estradiol ratios. Fertil Steril 2012; 98: 48-51.

3 Raman JD, Schlegel PN. Aromatase inhibitors for male infertility. J Urol 2002; 167: 624-9.

4 Cavallini G, Beretta G, Biagiotti G. Preliminary study of letrozole use for improving spermatogenesis in non-obstructive azoospermia patients with normal serum FSH. Asian J Androl 2011; 13: 895-7.

5 Patry G, Jarvi K, Grober ED, Lo KC. Use of the aromatase inhibitor letrozole to treat male infertility. Fertil Steril 2009; 92: 829. 
6 Saylam B, Efesoy O, Cayan S. The effect of aromatase inhibitor letrozole on body mass index, serum hormones, and sperm parameters in infertile men. Fertil Steril2011; 95: 809-11.

7 Beliveau ME, Turek PJ. The value of testicular 'mapping' in men with non-obstructive azoospermia. Asian J Androl 2011; 13: 225-30.

8 Koscinski I, Wittemer C, Lefebvre-Khalil V, Marcelli F, Defossez A et al. Optimal management of extreme oligozoospermia by an appropriate cryopreservation programme. Hum Reprod 2007; 22: 2679-84.

9 World Health Organization. WHO Laboratory the Examination and Processing of Human Semen. 5th ed. Geneva: World Health Organization; 2010.

10 Armitage P, Berry G, Mattews G. Statistical Methods in Medical Research. 4th ed. Oxford: Blackwell Science; 2002.

11 Ombelet W, Bosmans E, Janssen M, Cox A, Vlasselaer J et al. Semen parameters in a fertile versus subfertile population: a need for change the interpretation of semen testing. Hum Reprod 1997; 12: 987-93.

12 Tamhane AC, Mehta CR, Liu L. Testing a primary and a secondary endpoint in a group sequential design. Biometrics 2010; 66: 1174-84.

13 Turner L, Shamseer L, Altman DG, Schulz KF, Moher D. Does use of the CONSORT Statement impact the completeness of reporting of randomised controlled trials published in medical journals? A Cochrane review. Syst Rev 2012; 29: 60

14 Turkistani A, Marsh S. Pharmacogenomics of third-generation aromatase inhibitors. Expert Opin Pharmacother 2012; 13: 1299-307.

15 Carani C, Rochira V, Faustini-Fustini M, Balestrieri A, Granata AR. Role of oestrogen in male sexual behaviour: insights from the natural model of aromatase deficiency. Clin Endocrinol 1999; 51: 517-24.

16 Biagiotti G, Cavallini G, Modenini F, Vitali G, Gianaroli L. Spermatogenesis and spectral echo-colour Doppler traces from the main testicular artery. BJU Int 2002; 90: 903-8.

17 Donkol RH. Imaging in male-factor obstructive infertility. World J Radiol 2010; 2: 172-9.

18 Ma Y, Chen B, Wang H, Hu K, Huang Y. Prediction of sperm retrieval in men with nonobstructive azoospermia using artificial neural networks: leptin is a good assistant diagnostic marker. Hum Reprod 2011; 26: 294-8.
19 Jo B. Bias mechanisms in intention-to-treat analysis with data subject to treatment noncompliance and missing outcomes. J Educ Behav Stat 2007; 33: 158-85.

20 Hammoud AO, Griffin J, Meikle AW, Gibson M, Peterson CM et al. Association of aromatase [TTTAn] repeat polymorphism length and the relationship between obesity and decreased sperm concentration. Hum Reprod 2010; 25: 3146-51.

21 Chavarro JE, Toth TL, Wright DL, Meeker JD, Hauser R. Body mass index in relation to semen quality, sperm DNA integrity, and serum reproductive hormone levels among men attending an infertility clinic. Fertil Steril 2010; 93: 2222-31.

22 Schlegel PN. Aromatase inhibitors for male infertility. Fertil Steril 2012; 98: 1359-62.

23 Zhang Q, Bai Q, Yuan Y, Liu P, Qiao J. Assessment of seminal estradiol and testosterone levels as predictors of human spermatogenesis. J Androl 2010; 31 215-20.

24 Gennari L, Masi L, Merlotti D, Picariello L, Falchetti A et al. A polymorphic CYP19 TTTA repeat influences aromatase activity and estrogen levels in elderly men: effects on bone metabolism. J Clin Endocrinol Metab 2004; 89: 2803-10.

25 Meeker JD, Godfrey-Bailey L, Hauser R. Relationships between serum hormone levels and semen quality among men from an infertility clinic. J Androl2007; 28: 397-406.

26 Gordetsky J, van Wijngaarden E, O'Brien J. Redefining abnormal follicle-stimulating hormone in the male infertility population. BJU Int 2012; 110: 568-72.

27 Lotti F, Tamburrino L, Marchiani S, Muratori M, Corona G et al. Semen apoptotic M540 body levels correlate with testis abnormalities: a study in a cohort of infertile subjects. Hum Reprod 2012; 27: 3393-402.

28 Cavallini G, Magli MC, Crippa A, Resta S, Vitali G et al. The number of spermatozoa collected with testicular sperm extraction is a novel predictor of intracytoplasmic sperm injection outcome in non-obstructive azoospermic patients. Asian J Androl 2011; 13: 312-6.

29 La Sala GB, Nicoli A, Capodanno F, Rondini I, Villani MT et al. The effect of the 2004 Italian law on outcomes of assisted reproduction technology in severe male factor infertility. Reprod Biomed Online 2010; 20: 2-10.

30 Zanini G. Abandoned by the State, betrayed by the Church: Italian experiences of cross-border reproductive care. Reprod Biomed Online 2011; 23: 565-72.

31 Roy KK, Baruah J, Singla S, Sharma JB, Singh N et al. A prospective randomized tria comparing the efficacy of Letrozole and Clomiphene citrate in induction of ovulation in polycystic ovarian syndrome. J Hum Reprod Sci 2012; 5: 20-5. 